\title{
Are the evolutionary implications of vertical transmission of knowledge conservative?
}

\author{
Gustavo Taboada Soldati* and Ulysses Paulino Albuquerque ${ }^{2}$
}

\begin{abstract}
The evolution of cultural systems, or the rate of change in the frequency of traits, is determined by the routes of knowledge transmission, among other factors. According to mathematical models, vertical transmission is the more conservative route, and it promotes high variation among individuals of a population, acts as a barrier to the diffusion of innovations, and promotes slow cultural evolution. However, the history of transmission of the same cultural traits beyond "model-apprentice" pairs indicates that vertical transmission can produce different effects on a cultural system. In the present paper we formalize the hypothesis that vertical transmission has diffusive effects and results in a fast change cultural evolution. If the hypothesis proposed here is confirmed, the theoretical reformulation and relativization of empirical data collected in previous studies will be required.
\end{abstract}

Keywords: Social learning, cultural evolution, transmission of knowledge, Local ecological knowledge.

\section{INTRODUCTION}

A cultural system is a set of knowledge, beliefs, values, attitudes, skills and practices shared by a social group (Boyd and Richerson 2005). Because human behavior is essentially the performing of information (Mesoudi 2011), culture is the set of information (cultural traits) shared by social peers and acquired through different mechanisms of social learning (Boyd and Richerson 2005; Mesoudi 2011).

Various disciplines have attempted to describe "cultural evolution," or the mechanisms through which the frequency of a cultural trait varies in time and space (Mesoudi 2011). In the 1980s, two pairs of US researchers proposed a new theoretical framework for the study of cultural evolution based on mathematical models of population genetics and epidemiology. Cavalli-Sforza and Feldman (1981) and Boyd and Richerson (1985) founded their hypotheses on the assumption that the temporal and spatial dynamics of cultural systems occur according to Darwinian assumptions, such as mutation, competition and selection. They created a theoretical basis that includes micro and macroevolutionary processes in the context of cultural evolution. This new theoretical scenario allowed for the construction of formal models and hypotheses on the evolution of cultural systems.

Microevolutionary processes are responsible for variations in cultural traits at a small temporal and spatial scale usually within the same population (Mesoudi 2011). Examples include the modes and routes of knowledge transmission

1 Departamento de Botânica, Universidade Federal de Juiz de Fora, Rua José Lourenço Kelmer, s/n, Juiz de Fora, Minas Gerais. 36036 330. Brazil, gtsoldati@gmail.com

2 Laboratory of Ecology and Evolution of Social-Ecological Systems, Departamento de Biologia, Universidade Federal Rural de Pernambuco, Rua Dom Manoel de Medeiros, s/n, Dois Irmãos, Recife, Pernambuco. 52171-900. Brazil, upa677@hotmail.com 
and cultural selection. Over a long evolutionary period, these processes create macroevolutionary patterns, that is, at broader temporal and spatial scales, such as differences in culture among social groups and differences in the rate of cultural evolution (Mesoudi 2011).

One of the most frequently studied microevolutionary processes is the transmission of knowledge, and most studies apply the theoretical framework presented by Cavalli-Sforza and Feldman (1981), which defines the routes by which a cultural trait is transmitted among social peers and the evolutionary implications. According to these authors, a cultural trait can be transmitted socially through three routes: vertical, horizontal and oblique. Hewlett and Cavalli-Sforza (1986) restructured this original classification, subdividing the category "oblique" in two types: "one to many" and "many to one." Although not explicitly stated by the authors, this division is justified by the fact that these two new processes, which were previously grouped in the same category, have distinct evolutionary implications.

Hewlett and Cavalli-Sforza (1986) summarized these processes and stated that vertical transmission, which occurs between parents and offspring, promotes high variation in knowledge among individuals in a group and among different groups. This route is highly conservative because it hinders the diffusion of innovation; as a result, cultural evolution in a group that has adopted this type of strategy is quite slow. In systems that have well-developed horizontal routes, innovations are easily diffused. Groups in which transmission has dynamics similar to that of infectious diseases, knowledge variation among individuals and groups can be high and cultural evolution is rapid. The strategies "one to many" and "many to one" have distinct evolutionary implications. In the first case, the transfer occurs from a socially prominent person, such as a teacher, doctor or local expert, to a large group of individuals, usually pupils. Consequently, the diffusion of innovations occurs easily, and as a result of the homogenization process, such knowledge is similar among individuals within the group, despite the occasional high variation between groups (Hewlett and Cavalli-Sforza 1986). Similar to horizontal transmission, cultural evolution in groups that have adopted the strategy "one to many" proceeds relatively fast. The strategy "many to one" is also conservative because the values, traits and skills of the older members are transmitted at a large scale to other group members. This transmission channel inhibits the diffusion of innovation and knowledge diversification within the group and prohibits cultural evolution.

All of these theoretical assumptions originated from models that can simplify the transmission processes that occur in real cultural systems (see Henrich and Broesh 2011; Mace and Jordan 2011). Laboratory studies may indicate what occurs in natural systems, not just what happens (Mesoudi 2011). We started from the assumption that simplification may not always provide an accurate representation of the dynamics of the transmission processes in real systems. In the present study, we propose a hypothesis related to a microevolutionary process and its macroevolutionary implications. We reevaluated the relationship between the vertical transmission of knowledge and variation of cultural traits and cultural evolution. We hypothesized that when considering a broad history of transmission of a given cultural trait, i.e., additional transfer events, the evolutionary implications may be completely different from those forecasted by the theoretical models of Cavalli-Sforza and Feldman (1981), Boyd and Richerson (1985) and Hewlett and Cavalli-Sforza (1986). The majority of the empirical data on cultural evolution were recorded using "model-apprentice" pairs, such as "parent and child," which disregard the cultural history of the information transmitted, including its historical origin. The theoretical reflection that we propose is mediated by the analysis of traditional ecological knowledge systems, as we believe that these cognitive domains, because they are directly associated with the biological survival of the individual (see McElreath and Strimling 2008; Nairne and Pandeirada 2008), present rich adaptive information (see Takahashi 1998).

If our hypothesis is supported by the data collected in real cultural systems, a theoretical and conceptual reformulation will be required because such findings would indicate that the 
evolutionary implications of vertical transmission may not be conservative depending on how it occurs. Our proposal, if based on empirical data, do not represent "per se" an advance on existing models of cultural evolution, but would point weaknesses and needs of conceptual and theoretical adjustments.

\section{INTRODUCING THE HYPOTHESIS}

Several studies have sought to determine how transmission routes are represented in real cultural systems (e.g., Aunger 2000; Demps et al. 2012; Eyssartier et al. 2008; Hewlett et al. 2011; Hewlett and Cavalli-Sforza 1986; Lozada et al. 2006; Mathez-Stiefel and Vandebroek 2012; Reyes-Garcia et al. 2009; Tehrani and Collard 2009; Zarger and Stepp 2004). Apart from the existing methodological criticisms (see Aunger 2000; Essaytier et al. 2008; McElreath Strimling 2008; Henrich and Broesch 2011; Hewlett et al. 2011), these studies have suggested that social learning occurs throughout all life stages of the individual, although with different characteristics. Childhood is the stage during the greatest frequency of transmission events occurs, with the parental pairs (father, mother, grandfather, grandmother) of the apprentice being the most important models (Cavalli-Sforza et al. 1982; Hewlett and CavalliSforza 1986; Lozada and Ladio 2006; Setalaphruk and Price 2007).

The knowledge learned during childhood corresponds to basic and elementary information for the survival of the individual enculturation process (Aunger 2000; Hewlett and Cavalli-Sforza 1986), and it generally exhibits a more concrete and objective nature. Because of its nature, knowledge transmission can occur through simple observations or educational events. The name of the natural resource or instrument used on a daily basis is an example of information that can be transmitted. Although less frequent, social learning also occurs during adolescence and adulthood (see Aunger 2000; Cavalli-Sforza and Hewlett 1986; Demps 2012; Eyssartier et al. 2008; Hewlett et al. 2011). Because other non-parental models are available and accessed by the apprentices, other transmission routes are more predominant in these later stages, especially horizontal routes. In these stages of life, information is presented as updates of existing knowledge and exhibit a more tacit, subjective and complex nature that requires teaching and observation as well as implementation and experimentation by the apprentice (Aunger 2000). The management of natural resources, landscapes, or specific skills, such as hunting and honey gathering, are examples of this type of knowledge.

Thus, studies that consider the frequency of transmission events and are conducted in real systems indicate that vertical routes are the most important (e.g., Cavalli-Sforza et al. 1982; Hewlett and Cavalli-Sforza 1986; Lozada and Ladio 2006; Setalaphruk and Price 2007), although a consensus has not been found in the literature (see Aunger 2000; Reyes Garcia et al. 2009). Because of the theoretical assumptions developed on vertical transmission and cultural dynamics (see Hewlett and Cavalli-Sforza 1986), cultural systems are generally conservative and exhibit low rates of evolution and high levels of knowledge diversity among individuals of the same population. However, Aunger (2000) reconsidered the dynamics of vertical transmission by including the social origin of information that parents transfer to their offspring. Aunger suggests that "children may not learn what their parents learned when they were young (from grandparents), but instead what the parent more recently heard from someone outside the family." In addition, Aunger (2000) states that the evolutionary implications of the dynamics of vertical transmission are different from what is observed through mathematical predictions. Although the information is transmitted from parents to children, which is vertical, its social origin is in unrelated pairs. Aunger (2000) argues that diffusion of information may occur among representatives of the same group prior to vertical transmission. This specific dynamic transfer of traits could result in more homogeneous cultural systems despite being guided by the vertical routes, through a complex network of social exchanges. However, despite presenting a new understanding of the evolutionary implications of vertical transmission, Aunger (2000) did 
not formalize a hypothesis. Thus, based on Aunger's (2000) arguments, we formalized the following hypothesis to reassess the evolutionary implications of vertical transmission:

Assumptions: It is assumed that the information in a cultural system must be transmitted among different individuals and have a history of transmission that goes beyond a simple "modelapprentice" pair, even at the stage wherein this is expected to predominantly occur. Thus, it is possible that more than one transfer event of a given cultural trait may have resulted from different transmission routes. Thus, a vertical transmission event can provide at least two facets: a) conservative vertical transmission, which occurs when the trait that an individual learns has a history of parental transmission, indicating that it is internal to his/her family; and b) diffusive vertical transmission, which occurs when a trait that is transmitted from parents to children has an earlier origin in non-parental pairs.

Question: Is the vertical transmission of cultural traits purely conservative?

Hypothesis: The vertical transmission has conservative and diffusive events, which are predominantly diffusive.

Prediction: The frequency of cultural traits of diffusive origin is equal to or higher than the frequency of cultural traits of conservative origin.

To analyze the evolutionary implications of the hypothesis presented herein, we will present a typical cultural evolution model that assumes a population of individuals who each have a particular set of cultural traits. For heuristic purposes, each individual (sphere) in an ideal situation has six cultural traits (geometric shapes inside the spheres) (Figure 1A), which is based on the assumption that the access to information and its storage in memory systems cost energy that limits the amount of information learned. In this same ideal situation, we assume that of the six traits learned by an individual, four originate from the parents (solid geometric shapes), whereas the other two are copied from unrelated pairs (open geometric shapes), such as friends, teachers and local experts. However, the rate of vertical transmission in this model is always double the frequency of diffusive routes (horizontal, "many to one" and "one to many"). This prediction is used to simplify the present argument and finds resonance in the studies conducted in real systems that indicate vertical transmission as the predominant route (e.g., Cavalli-Sforza et al. 1982; Hewlett and Cavalli-Sforza 1986; Lozada and Ladio 2006; Setalaphruk and Price 2007). These same studies indicate that vertical transmission occurs preferentially during an individual's childhood (dark section of the sphere), whereas the diffusive routes are more common during adolescence and adulthood (light section of the sphere). As previously stated, the knowledge passed to children is of a different nature than the information passed to adults. With children, the information is preferentially objective, whereas with adolescents and adults, the information is generally more tacit. However, for analytical purposes, all traits present in the model are considered to have the same objective and practical nature, e.g., "knowledge on the therapeutic use of medicinal resources." This ability to retain concrete information learned during adulthood is recorded in different studies (see Aunger 2000; Soldati et al. 2015). 


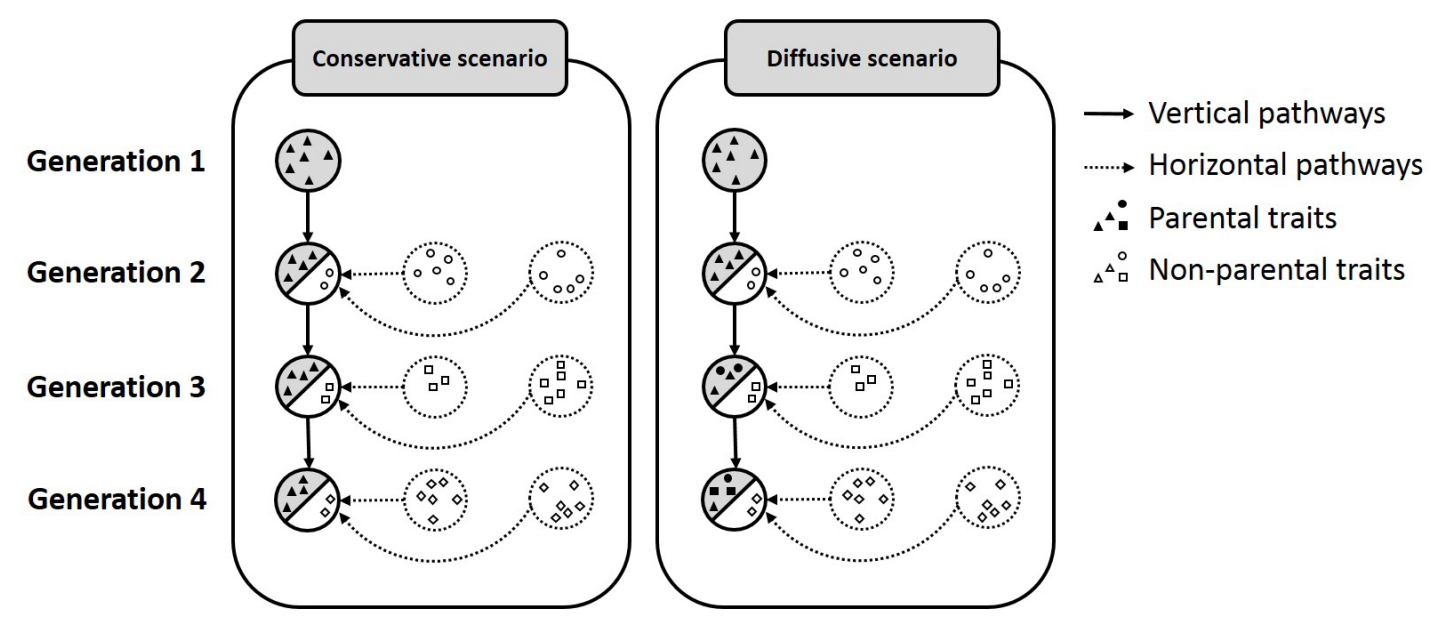

Fig 1. Visual representation of the cultural evolution model proposed in the present study that assesses cultural evolution based on two scenarios: conservative and diffusive. The evolutionary effects in each of the scenarios can be analyzed by the differences in cultural traits (geometric shapes) in fourth-generation individuals. In the conservative scenario, the information learned during childhood originates from vertical pairs, i.e., parents, grandparents, and great-grandparents. In the diffusive scenario, the cultural traits, although vertically transmitted, originate from non-parental pairs.

Although the frequency of vertical transmission is always double that of diffusive routes, the evolutionary implications of the cultural system can be completely different when considering the social origin of information from more than one transmission event of the same cultural trait, which extends beyond a single "model-apprentice" pair. To examine these implications, two distinct and extreme scenarios are considered, including a conservative and diffusive scenario (Figure 1B). In the first case, the four cultural traits learned by individuals during their childhood (dark section of the sphere) are the same traits that their parents learned from their grandparents, and so forth. Although learning occurs during adulthood through the incorporation of new information and new models, these new traits are not passed to the offspring, which is entirely plausible because biases that favor traits learned during childhood occur in the transmission of knowledge. For example, Soldati et al. (2015) observed that although adults acquire new knowledge of medicinal plants, they preferentially teach their children parental information because they consider such information more reliable. In the study by Soldati et al. (2015), informants more frequently used plants learned during childhood, thus resulting in higher transmission of these traits. From an evolutionary perspective, this behavior represents the understanding that "if I became sick in the past as a child but I am alive and healthy now, it is because the knowledge given to me by my parents is highly useful." Under this situation of extreme conservatism, the difference between knowledge carried by two individuals separated by four generations is of only two cultural traits of the six traits carried by each individual. Therefore, the rate of change/evolution (0.3333) is calculated as the total change divided by the total information (2/6).

Let us analyze again the diffusive scenario (Figure 1B) in which the traits learned during adulthood are necessarily transferred to the children. As we have shown, there is a limit to the information that can be stored in the memory systems; therefore, half of the parental origin traits under this extreme situation are replaced by nonparental information. There are also situations and biases that favor diffusive routes. The information learned during adulthood is an update of the 
individual's knowledge (Hewlett and Cavalli-Sforza 1986; Demps 2012); therefore, this information has the potential to provide additional benefit from an evolutionary perspective. In addition, situations of environmental instability favor the diffusive mechanisms of cultural transmission (McElreath and Strimling 2008; Reyes-Garcia et al. 2009). Under this diffusive scenario, the essentially vertical traits would be replaced by traits originated from non-parental pairs in only a few generations. Therefore, a comparison of the information between two individuals separated by four generations is qualitatively different. Of the six traits carried by the great-grandfather, none would be found in the great-grandson. The rate of change/evolution, calculated as the total change divided by the total information (6/6) is therefore equal to 1.0. Although vertical transmission is the most common transmission process, the evolutionary result in this cultural system would be the diffusion of information.

One can think that there is no reason to this discussion, cause no previous authors have claimed that a single cultural trait, by virtue of having been observed to have been passed down from parents to offspring, was necessarily also passed to the parents via the same route. But the fact that nobody has said this explicitly, not taken this into account, has the power to skew the micro and macroevolutionary interpretations. Also, one might think that our main question is not empirical but definitional because relative to other modes, independent of whether the traits being passed vertically in one generation were learned through other modes in previous generations. However, if we have a situation in which parents transmit to their children only learned traits learned of horizontal models, vertical transmission, in this case, will have exactly the same evolutionary implications of a horizontal transmission. So our question is empirical and conceptual.

\section{TESTING THE HYPOTHESIS}

To test the hypothesis proposed in the present study, the transmission route of a cultural trait prior to a vertical transmission event must be recorded; e.g., what model was used by a mother to learn the information that she taught her child. Thus, the basic information of analysis is the transfer of a given cultural trait. A cultural trait is understood to be a basic unit of cultural information capable of performing a behavior (see Mesoudi 2011). This study assumes that cultural traits are not categorical and immutable unit but minimally coherent so that they are not recognized and transmitted between social pairs, both spatially and temporally (Mesoudi 2011).

Therefore, informants would be parents (father, mother, grandmother or grandfather) of an apprentice. The sampling unit would not be a single informant but the pair formed by the apprentice (child informant) and one of his/her parental pairs (parent informant). After the selection of these pairs of informants, data collection would begin through information records of a particular cultural domain of local relevance and known by the "child informant," using a free listing (Albuquerque et al. 2014). To clarify our argument, a case study will be illustrated on the knowledge of medicinal plants. In this context, the cultural trait is not only the plant itself but the triad "plant-part-disease," which is the basic unit capable of producing a behavior. The free listing will allow access to known medicinal resources that were recorded by the "child informant" at the time of contact. The second step is to access the transmission routes of each resource, using semi-structured interviews. These tools can access the models used by the "child informant" during the learning process of the medicinal plants and their assigned uses. Because vertical transmission is the cultural process of interest, only traits arising from the parental pairs will be used for future analyses.

Subsequently, the "parental informant" will become the target of data collection. The triad "plant-plant part-disease" cited by the first informant for the vertical category should be presented to the "parental informant" to ensure that he/she understands the data. In cases with a negative response, the traits cited by the "child informant" should be excluded from the analysis because there is no way of determining which answer is true; thus, these data do not fall under the type of information needed for hypothesis testing, 
which includes information shared by parents and children and transmitted vertically. This situation may arise because the "child informant" is assigned to an event that did not actually occur. The same methodology used to access the transmission routes of the "child informant" should be applied to the "parental informant," considering only the traits shared between the two. This methodology allows for the past model of transmission of a given cultural trait to be recorded, thus following more than one event of cultural transfer of information. Therefore, it is possible to categorize the data for statistical comparisons. Because the hypothesis compares conservative and diffusive processes, two analytical categories are proposed: a) vertical transmission - conservative, which occurs when the trait is transmitted by vertical routes in both investigated events; and b) vertical transmission diffusive, which occurs when the previous origin of a trait, although transmitted vertically between "parental informant" and "child informant," previously originates in a non-parental model. To summarize, in the first proposed category, the learning route of the "parental informant" is vertical, whereas in the second category, corresponds to "horizontal" and "one to many" types, and they are both of a diffusive nature. Once the information collected from the informant pairs has been gathered, we can determine the frequency of each category using frequency tests (such as chi-square test, $\mathrm{G}$ test, etc.).

The data collection necessary to test the hypothesis focuses on self-reports information obtained, for example, by means of interviews. However, although this are the main tool for accessing information about the transmissions of knowledge (e.g. Arco et al. 2011; Eyssartier et al. 2008; Lozada et al. 2006; Srithi et al. 2009; Tehrani and Collard 2009;), this tool receives some criticism (see Aunger 2000; McElreath Strimling 2008; Hewlett et al. 2011; Henrich and Broesch 2011). It is argued that the informants tend to value the role of parents in the learning process, overestimating vertical routes (Aunger 2000; McElreath Strimling 2008; Hewlett et al. 2011; Henrich and Broesch 2011). To make more reliable data collection authors advocate combination with other methods, such as ethnographic descriptions or participant observations (see Aunger 2000). Reyes Garcia et al. (2009) sought to solve the fragility of semi structured interviews using correlational methods. We believe that the methods presented in this paper to test the hypothesis on the conservative and diffusive paths of vertical transmission also reflect a correlational approach, minimizing the effects of "self reportation", because a data will be included in the analyzes only when there is a correlation between citation of the child informant and parental informant in diachronic situations.

\section{IMPLICATIONS OF THE HYPOTHESIS}

The model of Cavalli-Sforza and Feldman (1981), which was reformulated by Hewlett and Cavalli-Sforza (1986), shows that in cultural systems in which the vertical transmission is the most important route, low acceptance for innovations will occur along with high knowledge variation among pairs and a low cultural evolution rate. However, the diffusive scenario presented here can visualize antagonistic evolutionary implications, thus skewing the implications of the cultural evolution theory. If the hypothesis proposed in the present study is confirmed, then theoretical reformulation and relativization of empirical data collected in previous studies will be required. For example, models of Cavalli-Sforza and Feldman (1981) and Boyd and Richerson (1985) assume that culture is usually adaptive and learning strategies have been shaped by natural selection. In this sense, it is assumed that unstable environments favor the individual production of knowledge (see Laland 2004) and the diffusive routes of transmission of knowledge (see McElreath and Strimling 2008; Reyes Garcia et al. 2009). Extrapolating these conditions to the discussion presented in this text, we expect the conservative scenario (Figure 1) occurs in relatively stable environments. However, Soldati et al. (2015) found, through empirical data, that situations of social environmental instability did not favor neither the individual production of knowledge or horizontal transmission routes. This study is therefore an example that in which the theoretical predictions not always occur in the empirical field. These premises, in the case 
of the knowledge of medicinal plants, are not true in reality investigated by Soldati et al. (2015). These actors used as analytical model the local knowledge about medicinal plants, because they considered that local ecological knowledge are directly linked to the individual's survival and provide better adaptive analysis (see also Takahashi 1998, Nairne and Pandeirada 2008). As the cultural selection (see Mesoudi 2011) favors different transmission mechanisms depending on the nature and content of cultural trait (McElreath and Strimling 2008), we expect therefore, that the diffuse vertical transmission discussed in this work is identified more often, for example, domains of local ecological knowledge.

Another important implication of this hypothesis is that support by empirical data requires a consideration of more than one transmission event of the same cultural trait during the data collection process. Although learning is an event that involves two social actors, the information itself has broader dynamics and an older cultural history. By considering the history of transmission of a given cultural trait, the cultural distribution of a trait can be determined by the relationship between a model and an apprentice as well as through different transmission processes and social actors, thus building a more complex network. Thus, a cybernetic approach can be used to better understand a cultural system through more systemic social models that consider the actors involved in a wider network consisting of different processes.

Finally, we emphasize the importance of the present hypothesis for the relationship among the routes of knowledge transmission and cultural system adaptability. Based on the understanding that only a biased transmission of information can promote cultural evolution, Henrich and Broesh (2011) emphasized the role of oblique transmission in the construction of cultural adaptations. According to these authors, the vertical routes are less flexible in terms of the "model-apprentice" pair, and the oblique routes enable the apprentice to choose the model and information to be absorbed. According to Henrich and Broesh (2011), this process increases cultural evolution through the conscious selection of traits and models recognized as more useful. Thus, the vertical route would be less flexible and consequently less adaptive in relation to this conscious selection. However, the present discussion confers to the vertical routes the same property discussed by Henrich and Broesh (2011) because even if learning is always fixed relative to the "model-apprentice" pair, the transferred information between parents and children is completely versatile. Thus, individuals teach what was learned from parents and what has been recognized as most beneficial. The only difference is that cultural selection within vertical routes is found in the model and not in the apprentice, which is inconsistent with oblique transmission.

Existing mathematical models (see Boyd and Richerson 1985; Cavalli-Sforza and Feldman 1981) assume that cultural transmission mechanisms have been shaped by natural and cultural selection, and is therefore adapted. In this sense, the environmental characteristics are important variables in the construction of behavior and learning strategies. For example, stable environments theoretically favor vertical routes as unstable environments stimulate the horizontal transmission types (see McElreath and Strimling 2008, Reyes-Garcia et al., 2009). However, our theoretical discussion has attempted to reflect on other mechanisms present in the transmission process influencing cultural developments, should parental selection of cultural information.

\section{REFERENCES}

1. Albuquerque UP, Ramos MA, Lucena RFP, Alencar NL (2014) Methods and techniques used to collect ethnobiological data. In: Albuquerque UP, Lucena RFP, Cunha LVFC, Ramos MA (orgs) Methods and techniques in Ethnobiology and Ethnoecology, 1 rd edn. Springer, New York, pp 15-37.

2. Aunger $R$ (2000) The life history of culture learning in a face-toface society. Ethos doi: 10.1525/eth.2000.28.3.445

3. Arco MPO, Torre-Cuadros MA, Reynel C (2011) Cultural transmission on Palms among Ese Eja communities in Peru. Bioremediation, Biodiversity and Bioavailability 5: 92-99.

4. Boyd R, Richerson PJ (1985) Culture and the evolutionary process. The University of Chicago Press, Chicago, USA.

5. Boyd R, Richerson PJ (2005) The origins and evolution of human culture. Oxford University Press, Oxford.

6. Cavalli-Sforza LL, Feldman M (1981) Cultural transmission and evolution: a quantitative approach, Princeton University Press, Princeton, USA. 
7. Demps K, Zorondo-Rodríguez F, García C, Reyes-García V (2012) Social learning across the life cycle: cultural knowledge acquisition for honey collection among the Jenu Kuruba, India. Evolution and Human Behavior 33. doi: 10.1016/j. evolhumbehav.2011.12.008

8. Eyssartier C, Ladio AH, Lozada M (2008) Cultural transmission of traditional knowledge in two populations of north-western Patagonia. Journal of Ethnobiology and Ethnomedicine 4. doi: 10.1186/1746-4269-4-25

9. Herich and Broesh (2011) On the nature of cultural transmission networks: evidence from Fijian villages for adaptive learning biases. Philosophical Transactions of the Royal Society B 366 . doi: 10.1098/rstb.2010.0323.

10. Hewlett BS, Cavali-Sforza LL (1986) Cultural transmission among Aka Pygmies. American Anthropologist 88: 922-934.

11. Hewlett BS, Fouts HN, Boyette AH, Hewlett BL (2011) Social Learning among Congo basin hunter-gatherers. Philosophical Transactions of the Royal Society B 366. doi: 10.1098/ rstb.2010.0373.

12. Laland, K. N. 2004. Social learning strategies. Learning \& Behavior 32: 4-14.

13. Lozada M, Ladio A, Weigandt M (2006) Cultural transmission of ethnobotanical knowledge in a rural community of northwestern Patagonia, Argentina. Economic Botany 60:374-385.

14. Mace R, Jordan F (2011) Macro-evolutionary studies of cultural diversity: a review of empirical studies of cultural transmission and cultural adaptation. Philosophical Transactions of the Royal Society B 366. doi:10.1098/rstb.2010.0238.

15. Mathez-Stiefel SL, Vandebroek I (2012) Distribution and transmission of medicinal plant knowledge in the Andean highlands: A case study from Peru and Bolivia. EvidenceBased Complementary and Alternative Medicine 2012: doi: 10.1155/2012/959285.

16. Mesoudi A (2011) Cultural Evolution: how darwinian theory can explain human culture, synthesize the social sciences. The University of Chicago Press, Chicago, USA.

17. McElreath $R$, Strimling $P(2008)$ When natural selection favors imitation of parents. Current Anthropology 49: 307-316.

18. Nairne JS, Pandeirada JNS (2008) Adaptive memory: is survival processing special? Journal of Memory and Language 59: 377385

19. Wataru Nakahashi \& Joe Yuichiro Wakano \& Joseph Henrich (2012) Adaptive Social Learning Strategies in Temporally and Spatially Varying Environments How Temporal vs. Spatial Variation, Number of Cultural Traits, and Costs of Learning Influence the Evolution of Conformist-Biased Transmission, Payoff-Biased Transmission, and Individual Learning. Human Nature 23:386-418.

20. Reyes-Garcia V, Molina JL, Broesch J, Calvet L, Fuentes-Pelaez N, McDade TW, Parsa S, Tanner S, Huanca T, Leonard WR, MartinezRodriguez MR (2009) Cultural transmission of ethnobotanical knowledge and skills: an empirical analysis from an amerindian society. Evolution and Human Behavior 30. doi: 10.1016/j. evolhumbehav.2009.02.001.

21. Setalaphruk C, Price LL (2007) Children's traditional ecological knowledge of wild food resources: a case study in a rural village in Northeast Thailand. Journal of Ethnobiology and Ethnomedicine 3. doi:10.1186/1746-4269-3-33

22. Soldati G T, Hanazaki N, Crivos M, Albuquerque UP (2015) Does environmental instability favor the production and horizontal transmission of knowledge regarding medicinal plants? A study in southeast Brazil. Plos One: 10(5) doi: 10.1371/journal. pone.0126389

23. Srithi $K$, Balslevb $H$, Wangpakapattanawonga $P$, Srisangac $P$, Trisonth C (2009) Medicinal plant knowledge and its erosion among the Mien (Yao) in northern Thailand. Journal of Ethnopharmacology 123 doi: 10.1016/j.jep.2009.02.035.

24. Takahasi, K. 1998. Evolution of Transmission Bias in Cultural Inheritance. Journal of Theoretical Biology 190: 147-159.

25. Tehrani JJ, Collard M (2009) On the relationship between interindividual cultural transmission and population-level cultural diversity: a case study of weaving in Iranian tribal populations. Evolution and Human Behavior 30 doi: 10.1016/j. evolhumbehav.2009.03.002.

26. Zarger RK, Stepp JR (2004) Persistence of botanical knowledge among Tzeltal Maya children. Current Anthropology 45: doi: 10.1086/420908.
Received: 02 February 2016

Accepted: 30 April 2016

Published: 30 June 2016 